\title{
Evolution Technique in Wireless Technology
}

\author{
Ganapathiram. $\mathbf{N}^{1}$, Sampath Kumar. $D^{2}$, Santhosh. $\mathbf{N}^{3}$ \\ Department of Computer Technology, Sri Krishna Arts \& Science College, Coimbatore ${ }^{1,2,3}$
}

\begin{abstract}
Fifth generation mobile technology expanded as $5 \mathrm{~g}$ technology. starting from generation $1 \mathrm{~g}$ to $2.5 \mathrm{~g}$ and $3 \mathrm{~g}$ to $5 \mathrm{~g}$. It has seen an great increase in technology. Based on mobile computing our day to day life changes. This paper displays increase in generation of mobile communication with 5th generation technology.5th generation network gives users affordable broadcast wireless connectivity also known as high speed connectivity. But the $5 \mathrm{~g}$ term is not legally used. $5 \mathrm{~g}$ is mainly used in development on worldwide wireless web.5th generation mainly targets on voice over IP(voIP)enable devices in which user can obtain high level call volume and data transmission.5th generation fulfill all needs which are required by the customers. The main target of $5 \mathrm{G}$ is the customer who want advanced feature in cellular phones. The main aim of $5 \mathrm{G}$ is to connect to multiple wireless technologies. This upgraded mobile technology will support IPV6 and flat IP. It provides services similar to documentation and e-transactions and e-payments.
\end{abstract}

Keywords: IPV6, 0G,1G, 2G, 2.5G, 3G, 4G, 5G, World Wide Wireless Web, WPANs, e-transactions and e-payments

\section{INTRODUCTION}

Wireless communication has seen an phenomenal growth in last few years it has increased from $1 \mathrm{G}$ to $5 \mathrm{G}$ generation. The main advantage of $5 \mathrm{G}$ is high bandwith which user never experienced before. It has more powerful features which makes $5 \mathrm{G}$ an huge demand in future. Wireless access technology evolution must start in fourth generation. $1^{\text {st }}$ generation had fulfilled basic mobile voice. Second generation consists of capacity and coverage. The main focus of $3^{\text {rd }}$ generation is high data rate and multimedia support. Fourth generation includes advanced mobile service with wide range of telecommunication. IP principle is implemented in all mobile networks. That says all data and signals will be transferred via IP(internet protocol). Camera ,mp3,recordings, video are provided in $5^{\text {th }}$ generation. $5^{\text {th }}$ generation technology provides enormous data capability and unrestricted call volumes and unlimited data broadcasting with latest operating system. $5^{\text {th }}$ generation should add more benefits and service over 4G.It should act as an path for connecting entire world.

\section{G (Zero Generation Mobile System):}

In 1940's, the first radio telephone service was introduced, Cars were designed to the users to the public land-line based telephone network. In the 1960's, Bell Systems, launched Improved Mobile Telephone Service (IMTS), made improvements such as direct dialing and more bandwidth. The first analog systems were based upon IMTS and were created in the $60 \mathrm{~s}$ and $70 \mathrm{~s}$.

First Generation(1G):

$1 G$ started in 1980s. It contains Analog System and known as cell phones. It introduces Mobile Telephone System (MTS) technology and Advanced Mobile Telephone System (AMTS), Improved Mobile Telephone Service (IMTS), and Push to Talk (PTT). It contains analog radio signal which have frequency $150 \mathrm{MHz}$, Frequency-Division Multiple Access (FDMA) is used for voice call. It has low capacity, poor voice links, without security voice calls were played back in radio tower, making these calls unwanted by third parties.

\section{Second Generation(2G):}

2G started in late 1980s. digital signals were used for voice transmission and has speed of 64 kbps. It provides SMS(Short Message Service) facilities and use the bandwidth of 30 to $200 \mathrm{KHz}$. second-generation (2G) systems used digital multiple access technology, such as TDMA (Time Division Multiple Access) and CDMA (Code Division Multiple Access). Consequently, better data services, and more advanced roaming were offered by $2 \mathrm{G}$ systems.

\subsection{G generation:}

It consists of 2G-systems that have implemented a packet switched domain in addition to the circuit switched domain. $2.5 \mathrm{G}$ data rate is up to $144 \mathrm{kbps}$. GPRS, EDGE and CDMA 2000 were used in 2.5 technologies.

\section{Third Generation(3G):}

3G used Wide Brand Wireless Network which had high clarity . Packet Switching technology is used for data transaction. Voice calls are interpreted through Circuit Switching. it provides data services, access to television/video, 
Global Roaming was introduced as new service. It operates at a range of $2100 \mathrm{MHz}$ and has a bandwidth of $15-20 \mathrm{MHz}$ used for High-speed internet service.3G uses Wide Band Voice Channel which made a person to contact with other person located in any part of the world and can send and recieve messages too.

\section{Fourth Generation(4G):}

4G provides downloading speed upto $100 \mathrm{Mbps} .4 \mathrm{G}$ has similar feature like $3 \mathrm{G}$ and additional services like MultiMedia Newspapers, to watch T.V programs with more clarity and send Data much faster than previous generations. 4G technology consists of LTE (Long Term Evolution). 4G is being developed mainly for wireless broadband access, Multimedia Messaging Service (MMS), video chat, mobile TV, HDTV content, Digital Video Broadcasting.

\section{WHAT IS 5G TECHNOLOGY ?}

Fifth generation of wireless mobile network which will begin in 2020s. It has almost no limitation which is huge advantage wireless communication. Mobile users does not had such a highly advance technology .An end user can also connect their desktops with $5 \mathrm{G}$ mobile phones to have internet connection. It totally supported World Wide Wireless Web (WWWW). This communication technology consists many benefits on mobile phones like dialing speed, MP3 recording, cloud storage, HD downloading will be more than the users imagination .

\section{Architecture of 5G:}

$5 \mathrm{~g}$ mobile system model is all-IP based model for wireless and mobile networks interoperability (AIPN) The All-IP Network is capable to improve increasing demands of the cellular communications market. It acts as an common platform for all radio access technologies. The AIPN uses packet switching and provides optimized performance. The $5 \mathrm{~g}$ Network Architecture consist of a user terminal (which has important role in the new architecture) and number of independent and autonomous radio access technologies (RAT). Cloud Computing Resources (CCR) provides IP based mobile applications and services such as Mobile portals, Mobile commerce, Mobile health care, Mobile government, Mobile banking and others, are offered via 5G Network Architecture . Cloud computing is a model for convenient ondemand network access to configurable computing resources (e.g., networks, servers, storage, applications, and services).with use of Cloud computing user can use applications without installation and also can access their data at any computer with internet access. CCR is linked with the Reconfigurable Multi Technology Core (RMTC) with remote reconfiguration data from RRD attached to (RDM) Reconfiguration Data models. The main task for a RMTC is to deal with increasing different radio access technologies. The core is a convergence of nanotechnology, cloud computing and radio, and based on All IP Platform. depending on status of the network and/or user demands communication function of core is changed. Other standards like IS/95, EV- DO, CDMA2000...etc are also enabled . Both terminal and RMTC are enabled using Interoperability process-criteria and mechanisms to select from heterogeneous access systems.

\section{WWWW (World Wide WirelessWeb)}

Wireless-based web applications that include full multimedia capability beyond 4G speed. The wireless Web refers the use of World Wide Web through a wireless device, such as a cellular telephone or (PDA). personal digital assistant . The e-mail, mobile banking, instant messaging, weather and travel information, and other services contains Wireless Web connection which provides anytime/anywhere connection. Sites which are aiming to accommodate wireless users must provide services in a format displayable on typically small wireless devices. It is calculated that $95 \%$ of wireless Internet devices being manufactured today. Use the Wireless Application Protocol (WAP) Motorola, Nokia, and Unwired Planet (now Phone.com) for presenting content. The wireless Web is not gaining in popularity as fast as some have predicted. The low bandwidth of today's wireless service and relatively high usage charges, and small and difficult-to-use input and output devices contribute to impeding growth.WWWW that is World Wide Wireless Web,which allows complete wireless communication with no limitation, Multi-Media Newspapers, watch TV programs with the clarity as to that of an HD TV. To enjoy this technology, mobile hardware must improve and should provide larger phone memory, quicker dialing speed, more clarity in audio and video etc $.4 \mathrm{G}$ technologies is the root cause for WWWW, World Wide Wireless Web. The following evolution is based up on $4 \mathrm{G}$ and completed its idea to form a REAL wireless world. 5G should make an major difference and add more services and benefit to the world more than 4G. 5G must be a more intelligent technology that interconnects the entire world without limits.5G will be the completed version of WWWW, World Wide Wireless Web, which form a real wireless world with no more limitation with access.

\section{Hardware OF 5G:}

UWB Networks: higher bandwidth at low energy levels. This short-range radio technology is standard for wireless personal area networks (WPANs). UWB provides the needed cost-effective, power-efficient, high bandwidth solution for relaying data from host devices to devices in the area (up to 10 meters or 30 feet).

1) Bandwidth: 4000 megabits per second, which is 400 times faster than today's wireless networks. 
Vol. 7, Issue 11, November 2018

2) Smart antennas.

3)Multiplexing: CDMA (Code Division Multiple Access) CDMA employs analog-to-digital conversion (ADC) in combination with spread spectrum technology. Audio input is first digitized into binary elements. The frequency of the transmitted signal varies according to a defined pattern (code), so it can be intercepted only by a receiver whose frequency response is programmed with the same code.

\section{Software of $5 \mathrm{G}$ :}

1)5G will be unified standard of different wireless networks, including LAN technologies, LAN/WAN, WWWW World Wide Wireless Web, unified IP \& seamless combination of broadband.

2) (SDR) Software defined radio, Packet layer, Encryption, Flexibility and Anti-Virus.

\section{Key Concepts of 5G :}

1. Real wireless world with no more limitation

2. Wearable devices with AI capabilities.

3. Internet protocol version 6 (IPv6), where a visiting care-of mobile IP address is assigned according to location and connected network.

4. One unified global standard.

5. The user can continuously get connected to several wireless technologies and they can also move between them

6. 5G aims at providing ease of services to the end users at high speed. The applications developed mainly for minimizing the interaction between the application and the user.

7. Access technologies can be $2.5 \mathrm{G}, 3 \mathrm{G}, 4 \mathrm{G}$ or $5 \mathrm{G}$ mobile networks, Wi-Fi, WPAN or any other future access technology. In $5 \mathrm{G}$ the concept may be developed into multiple concurrent data transfer paths.

8. Cognitive radio technology, also known as smart-radio which allows different radio technologies to share the same spectrum efficiently by adaptively finding unused spectrum and adapting the transmission scheme to the requirements of the technologies.

9. (HAPS) The High altitude stratospheric platform station systems.

10. World Wide wireless web (WWWW),comprehensive wireless-based web applications which include full multimedia capability beyond 4G speed.

11. Dynamic Ad hoc Wireless Networks (DAWN) similarly identical to Mobile ad hoc network (MANET), wireless grids are combined with smart antennas, cooperative diversity and flexible modulation.

12.A main issue in beyond $4 \mathrm{G}$ systems is to make the high bit rates available in a larger portion of the cell, specifically to users in an exposed position in between several base stations.

13.In current research, this issue is addressed by cellular repeaters and macro-diversity techniques also called as group cooperative relay,

\section{Quality of Service(QOS)}

(NGN) Next Generation Networks consists of support functionalities for data transport and control transport, as well as functionalities for support of services and applications. The measurement of traffic is a basic control activity which provide Quality of Service . In addition 5G communication system is designed by the best Quality of Service (QoS). Quality of Service (QoS) refers to a network's ability to produce maximum bandwidth and deal with other network performance elements such as latency, error rate and uptime. Quality of service controls and manages network resources by setting priorities for specific types of data (video, audio, files) on the network. QoS is mainly applied to network traffic generated for video on demand, IPTV, VoIP, streaming media, videoconferencing and online gaming. The main goal of quality of service is to provide priority to networks, including dedicated bandwidth, controlled jitter, low latency and improved loss characteristics. Its technologies supply the elemental building blocks which is used for future business applications in campus, wide area networks and service provider networks. There are 3 fundamental components for basic QoS implementation : • Identification and marking techniques for coordinating QoS from end to end between network elements. - QoS within a single network element.. • To control and administer end-to-end traffic across a network QoS policy management and accounting functions are used.

\section{Threats on 5g Implementation :}

The following threats are expected from the $5 \mathrm{G}$ network as a future system.

i) All the network operators and service providers would share a common core network infrastructure, compromise of a single operator will lead to the complete collapse of the entire network infrastructure..

ii) Third-parties can masquerade as the users resulting in theft of service and billing frauds can easily happen.

iii) The 5G is a secure IP based solution so it will be vulnerable to all the security threats as the current Internet world.

iv) The serious threats are email-spam, the Spam over Internet telephony (SPIT), the new spam over VoIP

v) Misdirected communication and internet banking related frauds is lead by spooling attacks..

vi) Interception of private communications and Eavesdropping.

vii) Attacks like Phishing attacks, stealing bank account details and other secured information can happen. 


\section{IJARCCE}

\section{CONCLUSION}

Mobiles have become more important part in our day to day life. Current development is the outcome of various generations. This paper shows overall introduction to $5 \mathrm{G}$ technologies, main concepts of $5 \mathrm{G}$ technology, and advantage of 5G technology, applications, and wireless network architecture for 5G wireless technologies. The new coming 5G technology is available in the market in affordable rates, high future and much reliability than $4 \mathrm{G}$ technologies. Fifth generation technologies provides tremendous data capabilities and unrestricted call volumes. Fifth generation will make an important difference and add more services and benefits than 4G. Fifth generation will be more intelligent technology that interconnects the entire world without limits. In 2020 this technology can be implemented. The world of universal, uninterrupted access to information, entertainment with communication will open new dimension to our lives and change our life style significantly.

\section{REFERENCES}

[1]. Nascimento, Andrea, et al. "A characterization of mobility management in user-centric networks", Smart Spaces and Next Generation Wired/Wireless Networking. Springer Berlin Heidelberg, pg.314-325, 2013.

[2]. Akhilesh Kumar Pachauri,Ompal Singh,"5G Technology-Redefining wireless Communication in upcoming years", International Journal of Computer Science and Management Research Vol 1 Issue 1 Aug 2012.

[3]. PankajSharm "Evolution of Mobile Wireless Communication Networks-1G to 5G as well as Future Prospective of Next Generation Communication Network", International Journal of Computer Science and Mobile Computing, Vol.2, Issue. , pg.47 - 53,2013.

[4]. Reshma S. Sapakal,SonaliS.Kadam, "5G Mobile Technology", 1323 International Journal of Advanced Research in Computer Engineering \& Technology (IJARCET) Volume 2, Issue 2, pg.568,2013.

[5]. Santhi, K. R., et al., "Goals of true broad band's wireless next wave (4G-5G)" Vehicular Technology Conference, 2003. VTC 2003-Fall.2003 IEEE 58th.Vol.4.IEEE, 2003.

[6]. EricSavitz,Forbes,http://www.forbes.com/sites/ericsavitz/2012/10/22/gartner-10-critical-tech-trends-for-the-nextfive-years/, 2012.

[7]. UpadhyayNiki "5GWIRELESS TECHNOLOGY”, Gandhinagar Institute of Technology, Gujarat, India.

[8]. kaviarasanindia.files.wordpress.com/2010/06/4g-technology.ppt.

[9]. Mohd. MaroofSiddiqui "Vision of 5G Communication", A. Mantri et al.(Eds.): HPAGC 2011, CCIS 169, CSpringer-Verlag Berlin Heidelberg, pp. 252-256, 2011.

[10]. Mousa, A. M. (2012). "Prospective of Fifth Generation Mobile communications". International Journal of Next - Generation Networks (IJNGN) 4(3): 1-30.

[11]. Mishra, A."Fundamentals of Cellular Network Planning and Optimisation.”John Wiley \& Sons2004.

[12]. UMTS World "UMTS/3G History and Future Milestones",[Online]Available: http://www.umtsworld.com/umts/history.html, (2009).

[13]. Naik, G., Aigal, V., Sehgal, P. and Poojary, J. "Challenges in the implementation of Fourth Generation Wireless Systems". International Journal of Engineering Research and Applications (IJERA) 2 (2)L: 1353-1355. (2012).

[14]. Parikh, J. and Basu, A.“LTE Advanced: The 4G Mobile Broadband Technology"International Journal of Computer Applications 13 (5):17-21.

[15]. 3GPP TR 36.814, V9.0.0, 2010, Further Advancements for E-UTRA Physical Layer Aspects, March 2010.

[16]. Kumaravel K, "Comparative Study of 3G and 4G in Mobile Technology" International Journal of Computer Science 2011.

[17]. Jay R Churi, T Sudish, Surendran, Shreyas, Ajay Tugdi "Evolution of Network" International Conference in Advance in Communication and Computing Technologies 2012.

[18]. http://en.wikipedia.org/wiki/5G]

[19]. http://searchmobilecomputing.techtarget.com/definition/wireless-Web.

[20]. http://selise.ch/5g-mobile-technology 